\title{
THE ROTATION THEOREM FOR STARLIKE UNIVALENT FUNCTIONS
}

A. W. GOODMAN

1. Introduction. Let $S$ denote the class of functions

$$
F(z)=z+\sum_{n=1}^{\infty} a_{n} z^{n}
$$

which are regular and univalent in $|z|<1$, and map each circle $|z|$ $\leqq r<1$ onto a region starlike with respect to the origin. Let $\Sigma$ denote the class of functions

$$
\Phi(\zeta)=\zeta+\sum_{n=0}^{\infty} b_{n} \zeta^{n}
$$

which are regular, except for the simple pole, and univalent in $|\zeta|>1$ and map each circle $|\zeta| \geqq \rho>1$ onto a region whose complement is starlike with respect to the origin.

In this paper we shall find upper bounds for

$$
R_{1}=R_{1}(z)=\arg F^{\prime}(z), \quad R_{2}=R_{2}(\zeta)=\arg \Phi^{\prime}(\zeta),
$$

and

(1.4) $T_{1}=T_{1}(z)=\arg F(z)-\arg z, T_{2}=T_{2}(\zeta)=\arg \Phi(\zeta)-\arg \zeta$ for $F(z) \in S$ and $\Phi(\zeta) \in \Sigma$. For $R_{1}, T_{1}$, and $T_{2}$ the bounds obtained will be sharp.

The problem of finding the sharp upper bound for $R_{1}$ was first attacked by Bieberbach [2] ${ }^{1}$ who obtained the estimate

$$
\left|R_{1}(z)\right| \leqq 2 \arcsin r+\arcsin \frac{2 r}{1+r^{2}}, \quad|z|=r,
$$

which is sharp only in the limit as $r \rightarrow 1$. Stroganoff [8] proved that the sharp bound for $R_{1}$ is attained for a function of the form $z /(1-z)^{2}$ for an appropriate value of $z$, but his proof is rather long and complicated. In $\$ 2$ we shall present a proof of this result, which is both shorter and simpler than the one given by Stroganoff.

Birnbaum [3] obtained upper bounds for $R_{2}$ and $T_{2}$. In $\$ 3$ we obtain the sharp bound for $T_{2}$, and an improved bound for $R_{2}$, and our method brings to light the difficulty of finding the sharp bound

Presented to the Society, September 5, 1952; received by the editors June 9, 1952.

${ }^{1}$ Numbers in brackets refer to the bibliography at the end of the paper. 
for $R_{2}$. The sharp bound for $T_{1}$ is easy to obtain, and is added for completeness.

Finally, we note that Urazbaev [9] has extended Stroganoff's proof to the subclass of $S$, of functions which are $k$-wise symmetric with respect to the origin. In $\$ 4$ we note that our method is equally valid for this subclass, and we state the result without details.

Bounds for $R_{j}$ and $T_{j}$ are of some geometric interest, since $R_{j}$ is the rotation of a lineal element at $z$ under the transformation, and $T_{j}$ is the angular rotation, under the transformation, of the point $z$ as viewed from the origin.

2. The bound for $R_{1}$. We begin with two elementary lemmas.

LemMA 1. Let $u_{1}, u_{2}, v_{1}, v_{2}, \alpha_{1}, \alpha_{2}$ be positive and such that $u_{1}<u_{2}$. Then the inequalities

$$
\frac{v_{1}}{u_{1}} \leqq \frac{v_{2}}{u_{2}}
$$

and

$$
\frac{\alpha_{1} v_{1} / u_{1}+\alpha_{2} v_{2} / u_{2}}{\alpha_{1}+\alpha_{2}} \leqq \frac{\alpha_{1} v_{1}+\alpha_{2} v_{2}}{\alpha_{1} u_{1}+\alpha_{2} u_{2}}
$$

each imply the other, and the equality sign occurs in (2.1) if and only if it occurs in (2.2).

Lемма 2. Suppose that for the positive quantities $\alpha_{j}, u_{j}, v_{j}, j$ $=1,2, \cdots, n$, the following conditions are satisfied:

$$
\begin{array}{ll}
\frac{v_{1}}{u_{1}} \geqq \frac{v_{j}}{u_{j}}, \quad v_{1} \leqq v_{j}, & j=2,3, \cdots, n, \\
u_{j}>1 / 2, & j=1,2,3, \cdots, n,
\end{array}
$$

and

$$
\sum_{j=1}^{n} \alpha_{j}=2
$$

Then

$$
\frac{\sum_{j=1}^{n} \alpha_{j} v_{j}}{\left(\sum_{j=1}^{n} \alpha_{j} u_{j}\right)-1} \leqq \frac{2 v_{1}}{2 u_{1}-1}
$$


and the equality sign occurs in (2.6) only if it occurs in both parts of (2.3) for all indices $j$.

The proofs of these two lemmas are direct and simple and we omit them.

Robertson $[7$, p. 376] has proved that if $f(z) \in C$, that is, if $f(z)$ $=z+\cdots$ is regular and univalent in $|z|<1$, and maps $|z|<1$ onto a convex region, then there exists a sequence of functions $f_{n}(z)$, of the form ${ }^{2}$

$$
f_{n}(z)=\int_{0}^{z} \prod_{j=1}^{n}\left(1-t e^{i \theta_{j}}\right)^{-\alpha_{j}} d t
$$

where $\theta_{j}$ is real, $\alpha_{j}>0, j=1,2, \cdots, n$, and $\alpha_{1}+\alpha_{2}+\cdots+\alpha_{n}=2$, such that $f_{n}(z) \rightarrow f(z)$, the convergence being uniform in any closed circle $|z| \leqq r<1$.

By Alexander's theorem [1] if $f(z) \in C$, then $F(z)=z f^{\prime}(z) \in S$, and conversely by integrating such an expression, if $F(z) \in S$, then $f(z)$ $\in C$. To obtain bounds for $R_{1}$ it is therefore sufficient to examine functions of the form

$$
F(z)=\frac{z}{\prod_{j=1}^{n}\left(1-e^{\left.i \theta_{j} z\right)^{\alpha_{i}}}\right.}
$$

subject to the same conditions as (2.7). An easy computation, using (2.8), (2.5), and the fact that $\arg F^{\prime}(z)=\Im \log F^{\prime}(z)$, yields

$$
R_{1}(z) \doteq \sum_{j=1}^{n} \alpha_{j} \Im \log \frac{1}{1-z e^{i \theta_{j}}}+\Im \log \left(\sum_{j=1}^{n} \frac{\alpha_{j}}{1-z e^{i \theta_{j}}}-1\right) .
$$

We next observe that it is sufficient to consider (2.9) only for $0<z<1$. For if $z_{0}=r e^{i \theta_{0}}$ is a point at which $\arg F^{\prime}(z)$ is a maximum, then $F_{1}(z)$ $=e^{-i \theta_{0}} F\left(z e^{i \theta 0}\right) \in S$, and $F_{1}^{\prime}(r)=F^{\prime}\left(r e^{i \theta 0}\right)$. The problem, then, is to determine the variables $n, \theta_{j}, \alpha_{j}$, subject to the restrictions already made, so that for fixed $r$

$$
R_{1}(r)=\sum_{j=1}^{n} \alpha_{j} \Im \log \frac{1}{1-r e^{i \theta_{j}}}+\Im \log \left(\sum_{j=1}^{n} \frac{\alpha_{j}}{1-r e^{i \theta_{j}}}-1\right)
$$

is a maximum.

Now the function $w=(1-r z)^{-1}=u+i v$ maps $|z| \leqq 1$ onto a circle $K$ in the $w$-plane with diameter end points $1 /(1-r)$ and $1 /(1+r)$

2 This is of course the Schwarz-Christoffel transformation, and it has frequently been used as the basis of investigations. For further references see [5]. 
$>1 / 2$. Setting $w\left(e^{i \theta j}\right)=u_{j}+i v_{j}$, equation (2.10) becomes

$$
R_{1}(r)=\sum_{j=1}^{n} \alpha_{j} \arctan \frac{v_{j}}{u_{j}}+\arctan \frac{\sum_{j=1}^{n} \alpha_{j} v_{j}}{\left(\sum_{j=1}^{n} \alpha_{j} u_{j}\right)-1}=A+B
$$

Since the circle $K$ lies entirely in the half-plane $\Re(w)>1 / 2$, and since the $\log$ functions in (16) are determined by the requirement $\log 1=0$, it follows that in (2.11) the arc tan function lies between $-\pi / 2$ and $\pi / 2$.

The points $w_{j}=u_{j}+i v_{j}$ lie on the boundary of $K$, and it is clear that to maximize $R_{1}(r)$ given by $(2.11)$ one should first take $v_{j}>0$ for $j=1,2, \cdots, n$. Secondly, if for some $j, u_{j}>1 /\left(1-r^{2}\right)$, the center of $K$, then $w_{j}$ can be replaced by a point for which $v_{j}$ is the same but $u_{j}<1 /\left(1-r^{2}\right)$, and both $A$ and $B$ will be increased by this change. We can now number the distinct points $w_{j}$ so that $1 / 2<u_{1}<u_{2}<\ldots$. $<u_{n}$ and, since the points are on the boundary of $K, 0<v_{1}<v_{2}<\ldots$ $\left\langle v_{n}\right.$. We form the ratios of $v_{j} / u_{j}, j=1,2, \cdots, n$, and note that either the inequality (2.1) of Lemma 1 holds, or the inequalities (2.3) of Lemma 2.

If the inequality (2.1) occurs, we leave unaltered the points $w_{j}, j=3,4, \cdots, n$, and their associated $\theta_{j}, \alpha_{j}$, and we replace $w_{1}$ and $w_{2}$ by the point $w_{12}=\alpha_{1} u_{1}+\alpha_{2} u_{2}+i\left(\alpha_{1} v_{1}+\alpha_{2} v_{2}\right)$ which lies inside $K$, and associate with this point $\alpha_{12}=\alpha_{1}+\alpha_{2}$. Since $\operatorname{arc} \tan q$ is convex downward for $q>0$,

$$
\begin{aligned}
& \alpha_{1} \arctan \frac{v_{1}}{u_{1}}+\alpha_{2} \operatorname{arc} \tan \frac{v_{2}}{u_{2}} \\
&<\left(\alpha_{1}+\alpha_{2}\right) \arctan \frac{\alpha_{1} v_{1} / u_{1}+\alpha_{2} v_{2} / u_{2}}{\alpha_{1}+\alpha_{2}} .
\end{aligned}
$$

Combining this with the inequality (2.2), it follows that, in (2.11), $A$ is increased by the replacement of $w_{1}$ and $w_{2}$ by $w_{12}$, and it is obvious that $B$ is unchanged. We may now replace $w_{12}$ by a point on the boundary of $K$ leaving $u_{12}$ fixed but increasing $v_{12}$, and this replacement increases both $A$ and $B$. This point determines a $\theta_{12}$ which with $\alpha_{12}$ determines a new function $F(z)$ of the form (2.8) with only $n-1$ terms in the product, and for which $R_{1}(r)$ is larger than for the original $F(z)$. This process may be repeated, and after a finite number of such steps, we have either an $F(z)$ of the form (2.8) with only one term in the denominator, or we arrive at an $F(z)$ for which all the 
conditions of Lemma 2 are satisfied, with inequality in (2.3).

If the conditions of Lemma 2 are satisfied, replace $w_{j}$ by $w_{1}$ for $j=2,3, \cdots, n$, and $\alpha_{1}$ by $\alpha_{1}+\alpha_{2}+\cdots+\alpha_{n}$. It is clear from inequality (2.3) that $A$ is increased, and from inequality (2.6) that $B$ is increased. This completes the proof that $R_{1}(r)$ assumes its maximum for a function of form $F(z)=z /\left(1-z e^{i \theta}\right)^{2}$. Direct computation, or a simplification of (2.11), yields, for this function,

$$
R_{1}(r)=\arctan \frac{r \sin \theta}{1+r \cos \theta}+3 \arctan \frac{r \sin \theta}{1-r \cos \theta} .
$$

The extreme values occur in (2.13) for the roots of

$$
Q(\cos \theta)=2 r \cos ^{2} \theta+2\left(1-r^{2}\right) \cos \theta-r\left(1+r^{2}\right) .
$$

Since $Q(-1)=(r-2)\left(1-r^{2}\right)<0, Q(0)<0$, and $Q(1)=(2+r)\left(1-r^{2}\right)$ $>0$, the extreme values of $R_{1}(r)$ are obtained for $\theta=\theta_{0}$ where

$$
\cos \theta_{0}=\frac{r^{2}-1+\left(1+3 r^{4}\right)^{1 / 2}}{2 r} .
$$

Either the symmetry of the problem, or the fact that $R_{1}(r)$ is an odd function of $\theta$, shows that the maximum corresponds to the first quadrant angle and the minimum corresponds to the fourth quadrant angle given by (2.15).

Theorem 1. If $F(z) \in S$, and $0<|z|=r<1$, then

$$
\left|\arg F^{\prime}(z)\right| \leqq \arctan \frac{r \sin \theta_{0}}{1+r \cos \theta_{0}}+3 \arctan \frac{r \sin \theta_{0}}{1-r \cos \theta_{0}}
$$

where $0<\theta_{0}<\pi / 2$ is given by (2.15). This bound is sharp for each $r$, equality occurring for $F_{M}(z)=z /(1-z)^{2}$ at $z=r e^{i \theta 0}$.

3. The bounds for $T_{1}, T_{2}$, and $R_{2}$. From equation (2.8) with $z=r e^{i \theta}$, we have

$$
T_{1}(z)=-\sum_{j=1}^{n} \alpha_{j} \arg \left(1-r e^{i\left(\theta+\theta_{j}\right)}\right) .
$$

For $|z| \leqq 1$, the points $w=(1-r z)$ fill a circle of radius $r$ and center at $w=1$, so that $|\arg (1-r z)| \leqq \operatorname{arc} \sin r$. This gives

THEOREM 2. If $F(z) \in S$, and $O<|z|=r<1$, then

$$
|\arg F(z)-\arg z| \leqq 2 \operatorname{arc} \sin r .
$$

This bound is sharp for each $r$, equality occurring for $F_{M}(z)$ at $z=r e^{i \theta_{1}}$, 
$\theta_{1}=\operatorname{arc} \cos r$.

It is well known, and easy to prove, that the elements of $S$ and $\Sigma$ are related by

$$
\Phi(\zeta)=\frac{1}{F(z)}, \quad z=\frac{1}{\zeta}
$$

and so $\Phi(\zeta) / \zeta=z / F(z)$. This gives immediately

THEOREM 3. If $\Phi(\zeta) \in \Sigma$, and $|\zeta|=\rho>1$, then

$$
|\arg \Phi(\zeta)-\arg \zeta| \leqq 2 \arcsin \rho^{-1} .
$$

This bound is sharp for every $\rho>1$, equality occurring for $\Phi_{M}(\zeta)$ $=\zeta-2+\zeta^{-1}$.

Birnbaum [3] obtained $2 \arcsin \rho^{-1}+\pi / 2$ for the right side of (3.4).

From equations (3.3) and (2.8) it follows that for each $\Phi(\zeta) \in \Sigma$ there is a sequence of functions of the form

$$
\Phi_{n}(\zeta)=\zeta \prod_{j=1}^{n}\left(1-\frac{e^{i \theta_{j}}}{\zeta}\right)^{\alpha_{j}}
$$

where $\theta_{j}$ and $\alpha_{j}$ satisfy the same conditions as in equation (2.7), such that $\Phi_{n}(\zeta) \rightarrow \Phi(\zeta)$, the convergence being uniform in any ring $1<\rho_{1}$ $\leqq|\zeta| \leqq \rho_{2}<\infty$.

Just as for $R_{1}(r)$, it is sufficient to consider $\zeta=\rho>1$. Setting $\rho=1 / r$, a computation from (3.5) yields

$$
\begin{array}{r}
R_{2}(\rho)=\sum_{j=1}^{n} \alpha_{j} \Im \log \left(1-r e^{i \theta_{j}}\right)+\Im \log \left(\sum_{j=1}^{n} \frac{\alpha_{j}}{1-r e^{i \theta_{j}}}-1\right) \\
=\arctan \frac{\sum_{j=1}^{n} \alpha_{j} v_{j}}{\left(\sum_{j=1}^{n} \alpha_{j} u_{j}\right)-1}-\sum_{j=1}^{n} \alpha_{j} \arctan \frac{v_{j}}{u_{j}}=B-A,
\end{array}
$$

where the points $w_{j}=u_{j}+i v_{j}$ lie on the boundary of the same circle $K$ as in equation (2.11). Since

$$
\left|R_{2}(\rho)\right| \leqq|B|+|A|
$$

we have immediately, from the proof of Theorem 1, the following result.

TheOREM 4. If $\Phi(\zeta) \in \Sigma$, and $|\zeta|=\rho=1 / r>1$, then 
(3.8) $\left|\arg \Phi^{\prime}(\zeta)\right| \leqq \arctan \frac{\sin \theta_{0}}{\rho+\cos \theta_{0}}+3 \arctan \frac{\sin \theta_{0}}{\rho-\cos \theta_{0}}<\frac{3 \pi}{2}$,

where $0<\theta_{0}<\pi / 2$ is given by (2.15).

Obviously this result is not sharp for any value of $\rho>1$, since equality can occur in (3.7) only if $n=1$, and then both $A$ and $B$ will have the same sign. Indeed for the particular function $\Phi_{M}(\zeta)=\zeta$ $-2+\zeta^{-1}$

$$
\left|\arg \Phi_{M}^{\prime}(\zeta)\right| \leqq \arcsin \rho^{-2} \leqq \pi / 2 \text {. }
$$

Despite the attractive form of (3.9) it can easily be shown that the constant $3 \pi / 2$ in (3.8) cannot be replaced by any smaller constant valid for all $\rho>1$.

To prove this assertion, it is sufficient to consider the special function $\Phi(\zeta)$ which maps $|\zeta|>1$ onto the exterior of an arrow. By an arrow we mean the figure consisting of three line segments meeting at a common end point. For simplicity we suppose the common end point set at the origin, the other end points of the segments at $s_{1} e^{ \pm i(1-\gamma) \pi}$, and $-s_{2}, s_{j}>0$. Thus, the arrow is symmetrical about its shaft and the angle between the barbs and the shaft is $\gamma \pi$. The argument could now be concluded on the basis of known existence theorems, and the theory of functions which map onto variable domains, but we can avoid appealing to these results, since the formula is explicitly known $[4 ; 6]$ for such a special function $\Phi(\zeta)$. Indeed it is not difficult to see that if $0<\gamma<1$, and $0<\theta_{1}<\pi / 2$, then

$$
\Phi(\zeta)=\zeta\left(1-\frac{1}{\zeta}\right)^{2-2 \gamma}\left(1-\frac{2}{\zeta} \cos \theta_{1}+\frac{1}{\zeta^{2}}\right)^{\gamma}
$$

maps $|\zeta|>1$ onto the exterior of the arrow described above. The critical points corresponding to the free end points of the line segment are $\zeta=-1$, and $\zeta=e^{ \pm i \beta}$ where

$$
\cos \beta=\gamma+(1-\gamma) \cos \theta_{1}>0 .
$$

The lengths of the barb and the shaft are given by

$$
s_{1}=\left(1-\cos \theta_{1}\right)(2-2 \gamma)^{1-\gamma} \gamma^{\gamma}
$$

and

$$
s_{2}=2^{2-\gamma}\left(1+\cos \theta_{1}\right)^{\gamma} \text {. }
$$

As $\theta_{1} \rightarrow 0$ it is clear that for each fixed $\gamma>0 s_{1} \rightarrow 0$ and $s_{2} \rightarrow 4$, and 
since $\theta_{1}>\beta>0$, it follows that $\beta \rightarrow 0$. The smaller arc on $|\zeta|=1$ bounded by $e^{i \beta}$ and $e^{i \theta_{1}}$ is transformed by the function (3.10) into the under side of the upper barb, the mapping being conformal on the interior of the arc. Any radial line in the closed region $|\zeta| \geqq 1$ ending at a point of this arc is transformed by $\Phi(\zeta)$ into a curve ending on the under side of the upper barb and perpendicular to it. If $\beta<\theta<\theta_{1}$, then $\arg \Phi^{\prime}\left(e^{i \theta}\right)=3 \pi / 2-\gamma-\theta$. But $\gamma$ and $\theta_{1}$ can be taken arbitrarily small.

4. Starlike functions with symmetry about the origin. We denote by $S_{k}$ the subclass of $S$ of functions of the form

$$
F(z)=z+\sum_{n=1}^{\infty} a_{n} z^{n k+1}
$$

$k$ a positive integer. For this subclass equation (2.8) can be replaced by

$$
F(z)=\frac{z}{\prod_{j=1}^{n}\left(1-e^{i \theta_{j} z^{k}}\right)^{\alpha_{j}}}
$$

where now

$$
\sum_{j=1}^{n} \alpha_{j}=\frac{2}{k}
$$

Equation (2.11) becomes

(4.4) $\quad R_{1}(r)=\sum_{j=1}^{n} \alpha_{j} \arctan \frac{v_{j}}{u_{j}}+\arctan \frac{k \sum_{j=1}^{n} \alpha_{j} v_{j}}{\left(k \sum_{j=1}^{n} \alpha_{j} u_{j}\right)-1}$

where $w_{j}=u_{j}+i v_{j}$ lies on the boundary of the circle $\Gamma$ which is the image of $|z| \leqq 1$ under

$$
w=\left(1-r^{k} z\right)^{-1} \text {. }
$$

The same proof as before shows this is a maximum when all the $w_{j}$ 's are coincident.

ThEOREM 5. If $F(z) \in S_{k}$, and $0<|z|=r<1$, then

$$
|\arg F(z)-\arg z| \leqq \frac{2}{k} \arcsin r^{k}
$$


and

$$
\begin{aligned}
\left|\arg F^{\prime}(z)\right| \leqq & \arctan \frac{r^{k} \sin \theta_{0}}{1+r^{k} \cos \theta_{0}} \\
& +\frac{k+2}{k} \arctan \frac{r^{k} \cos \theta_{0}}{1-r^{k} \cos \theta_{0}},
\end{aligned}
$$

where $0<\theta_{0}<\pi / 2$ is given by

$$
\cos \theta_{0}=\frac{(k+1)\left(r^{2 k}-1\right)+\left((k+1)^{2}\left(1-r^{2 k}\right)^{2}+8 r^{2 k}\left(1+r^{2 k}\right)\right)^{1 / 2}}{4 r^{k}}
$$

and both the bounds (4.5) and (4.6) are sharp for all $r<1$.

\section{REFERENCES}

1. J. W. Alexander, Functions which map the interior of the unit circle upon simple regions, Ann. of Math. vol. 17 (1915) pp. 12-22.

2. L. Bieberbach, Aufstellung und Geweis des Drehungssatzes für schlichte konforme Abbildungen, Math. Zeit. vol. 4 (1919) pp. 295-305.

3. Z. W. Birnbaum, Beiträge zur Theorie der schlichten Funktionen, Studia Math. vol. 1 (1929) pp. 159-190.

4. C. Darwin, On some transformations involving elliptic functions, Philosophical Magazine (7) vol. 41 (1950) pp. 1-11.

5. A. W. Goodman, On the Schwarz-Christoffel transformation and p-valent functions, Trans. Amer. Math. Soc. vol. 68 (1950) pp. 204-223.

6. - Inaccessible boundary points, Proceedings of the American Mathematical Society vol. 3 (1952) pp. 742-750.

7. M. S. Robertson, On the theory of univalent functions, Ann. of Math. vol. 37 (1936) pp. 374-408.

8. W. G. Stroganoff, Über den arc $f^{\prime}(z)$ unter der Bedingung dass $f(z)$ die konforme Abbildung eines sternartigen Gebietes auf das innere des Einheitskreises der z-Ebene liefert, Trudy Mat. Inst. Steklova. vol. 5 (1934) pp. 247-258.

9. B. M. Urazbaev, On the argument of the derivative of a univalent star-shaped function, Izvestiya Akad. Nauk. Kazah. SSR. vol. 56 Ser. Mat. Meh. 2 (1948) pp. 102-121 (Russian).

UNIVERSITY OF KENTUCKY 